\title{
New Diagnoses Cover Venomous Toads and Exploding Spacecrafts
}

\author{
Michael Huckabee* \\ University of Nebraska Medical Center, Omaha, USA
}

*Corresponding author: Michael Huckabee, Director, University of Nebraska Medical Center, Division of Physician Assistant Education 984300 Nebraska Medical Center Omaha, NE 68198-4300, USA, Tel: 01-402-559-7953; Fax: 01-402-559-7996; E-mail: michael.huckabee@unmc.edu

Rec date: Apr 17, 2014, Acc date: Sep 29, 2014; Pub date: Oct 1, 2014

Copyright: (c) 2014 Huckabee M. This is an open-access article distributed under the terms of the Creative Commons Attribution License, which permits unrestricted use, distribution, and reproduction in any medium, provided the original author and source are credited.

\section{Editorial}

Many of us are shaking our heads as another deadline looms for health care reform (exhibiting diagnostic code Z734, inadequate social skills not elsewhere classified). October 1, 2015, is the newest date for conversion to a new classification for diseases used by virtually all medical practices for billing purposes. It impacts how medicine gets paid for what we do. For anyone employed in health care it has our attention with three letters and a number: ICD-10.

Let's back up. For the last generation the healthcare team organized mankind's ailments and aches using the ninth edition of the International Classification of Diseases (ICD). First designed to track mortality statistics, the World Health Organization (WHO) publishes this list which is used by analysts and insurance companies. The ninth edition was published in 1975 and has been updated regularly; however, it represents a paltry 14,600 codes.

In the 1990s WHO developed a new version, ICD-10, with over 68,000 codes, and the world has been gradually adopting the latest international language of disease. The US is one of a few countries dragging its feet (which may be due to being bitten by a venomous toad, code T6382). Australia first converted to ICD-10 in 1998, Canada followed in 2000, and China in 2002. The US has sought to postpone the conversion primarily due to the massive overhaul required of our computer-driven payment systems. The world has been patient long enough and to avoid applying code Z565 (uncongenial work environment), the US has promised no more delays. America will flip the switch on October 1 for the new classification system. No one gets paid after that date if they try to submit ICD-9 codes. It feels like the Y2K of medicine (where the code Z9912 may be needed, respirator dependence during a power failure).
What's new? Rather than 3 to 5 numbers, now the codes will begin with a letter followed by 3 to 6 characters. Similar to Facebook reorganizing how it tracks friends, the new system is more sensibly organized and offers more detail. While there are a number of new codes, most of the revisions allow better descriptions for various conditions related to existing diagnoses. For example, a separate code is used for the initial visit for a diagnosis, distinct from the code used for a subsequent encounter for the same diagnosis. That change alone accounts for thousands of new codes (such as V9733XD, sucked into jet engine, subsequent encounter).

The new version also supports burgeoning new technology, including 21 codes involving spacecrafts (e.g., V9545XA, spacecraft explosion injuring occupant). Greater exactness is provided, such as seven different codes detailing injuries sustained when falling off a toilet.

Before anyone jumps off a cliff (W15XXXA), we should understand that the revisions primarily apply to computerized coding. This will require training and software upgrades, but this is no cause for rash behavior (F16921, hallucinogen use, unspecified with intoxication with delirium). Major health insurance plans such as BlueCross/BlueShield, Aetna, Humana, and United Healthcare have all told the American Academy of Family Physicians that they are ready for ICD-10.

While all the diagnoses used here are found in the ICD-10, one diagnosis is missing: "tongue in cheek." ICD-11 is scheduled for release in 2017. We'll look for it there. 